\title{
Cycloserine-Cefoxitin Fructose Agar
}

National Cancer Institute

\section{Source}

National Cancer Institute. Cycloserine-Cefoxitin Fructose Agar. NCI Thesaurus. Code C150828.

A solid growth medium mainly consisting of proteose peptone, fructose, cycloserine, cefoxitin, fructose, and egg yolk, which is used primarily for the isolation and cultivation of Clostridium difficile. 